\title{
Comparison Shopping for a Gradient-Corrected Density Functional
}

\author{
JOHN P. PERDEW AND KIERON BURKE \\ Department of Physics and Quantum Theory Group, Tulane University, New Orleans, \\ Louisiana 70118
}

Received June 19, 1994; revised manuscript received September 5, 1994; accepted October 27, 1994

\begin{abstract}
Gradient corrections to the local spin density (LSD) approximation for the exchangecorrelation energy are making density functional theory as useful in quantum chemistry as it is in solid-state physics. But which of the many gradient-corrected density functionals should be preferred a priori? We make a graphical comparison of the gradient dependencies of some popular approximations, discussing the exact formal conditions which each obeys and identifying which conditions seem most important. For the exchange energy, there is little formal or practical reason to choose among the Perdew-Wang 86, Becke 88, or Perdew-Wang 91 functionals. But, for the correlation energy, the best formal properties are displayed by the nonempirical Pw91 correlation functional. Furthermore, the real-space foundation of PW91 yields an insight into the character of the gradient expansion which suggests that PW91 should work especially well for solids. Indeed, while improving dissociation energies over LSD, PW91 remains the most "local" of the gradient-corrected exchange-correlation functionals and, thus, the least likely to overcorrect the subtle errors of LSD for solids. To show that our analysis of spin-unpolarized functionals is sufficient, we also compute spin-polarization energies for atoms, finding PW91 values only slightly more negative than LSD values. (c) 1996 John Wiley \& Sons, Inc.
\end{abstract}

\section{Introduction}

$\mathbf{K}$ ohn and Sham [1] proved that the exact ground-state density and energy of a manyelectron system may be found through the selfconsistent solution of a single-particle problem like that of Hartree-Fock $(\mathrm{HF})$ theory, but with the exchange energy and potential replaced by the exchange-correlation energy $E_{x c}\left[n_{\uparrow}, n_{\downarrow}\right]$, a functional of the up- and down-spin electron densities, and its functional derivative, the exchange-correlation potential, $v_{x c, \sigma}(\mathbf{r})=\delta E_{x c} / \delta n_{\sigma}(\mathbf{r})[2-4]$. They also proposed the local spin density (LSD) approximation,

$$
E_{x c}^{\mathrm{LSD}}\left[n_{\uparrow}, n_{\downarrow}\right]=\int d^{3} m(\mathbf{r}) \epsilon_{x c}\left(n_{\uparrow}(\mathbf{r}), n_{\downarrow}(\mathbf{r})\right),
$$

where $n=n_{\uparrow}+n_{\downarrow}$ and $\epsilon_{x c}\left(n_{\uparrow}, n_{\downarrow}\right)$ is the 
TABLE 1

Atomization energies of molecules (eV).

\begin{tabular}{lrrrr}
\hline Molecule & HF & \multicolumn{1}{c}{ LSD } & PW91 & Expt. \\
\hline $\mathrm{C}_{2}{ }^{\mathrm{a}}$ & 0.73 & 7.51 & 6.55 & 6.36 \\
$\mathrm{C}_{6} \mathrm{H}_{6}{ }^{\mathrm{a}}$ & 45.19 & 68.42 & 61.34 & 59.67 \\
$\mathrm{H}_{2}{ }^{\mathrm{b}}$ & 3.29 & 4.65 & 4.52 & 4.49 \\
$\mathrm{H}_{2} \mathrm{O}^{\mathrm{b}}$ & 5.71 & 11.00 & 9.59 & 9.51 \\
$\mathrm{O}_{2}{ }^{\mathrm{b}}$ & 1.25 & 7.48 & 5.93 & 5.12 \\
\hline
\end{tabular}

a From [19], using a basis set of 18 s-type, nine p-type, and four $d$-type single Gaussians on each atom. For $C_{2}$ and $\mathrm{C}_{6} \mathrm{H}_{6}$, the zero-point vibrational energy has been omitted from the calculated and experimental values.

bw91, using a triple-zeta valence plus polarization basis set, and Expt. are from [38]; HF, using a $6-31 \mathrm{G}^{*}$ basis, from [28]; and LSD, using a basis-free numerical method, from [23].

exchange-correlation energy per particle for an electron gas of uniform spin densities $n_{\uparrow}$ and $n_{\downarrow}$. This approximation has served solid-state physics well for almost 30 years.

More recently [5-16], generalized gradient approximations (GGAS), in which the exchangecorrelation energy is approximated by the form

$$
E_{x c}^{\mathrm{GGA}}\left[n_{\uparrow}, n_{\downarrow}\right]=\int d^{3} r f\left(n_{\uparrow}(\mathbf{r}), n_{\downarrow}(\mathbf{r}), \nabla n_{\uparrow}, \nabla n_{\downarrow}\right),
$$

have become popular in both quantum chemistry and condensed matter physics. They typically yield total energies [17-20] and atomization energies [21-31] that are more accurate than those of the HF or LSD approximations, as illustrated by Table I, as well as improved barriers to chemical reactions [32-34] and improved lengths of hydrogen [35-38] and metal-ligand [39] bonds. GGAs can also correct the more subtle LSD errors in the lattice constants and bulk moduli of solids, as illustrated in Table II.

The correct input $\epsilon_{x c}\left(n_{\uparrow}, n_{\downarrow}\right)$ which makes Eq. (1) exact in the limit of constant density is well known $[40,41]$, and this limit itself is important in the theory of crystalline metals. But there are many different prescriptions for the input

$$
f\left(n_{\uparrow}, n_{\downarrow}, \nabla n_{\uparrow}, \nabla n_{\downarrow}\right)
$$

to Eq. (2). The choice of a "best" GGA for all electronic systems requires an understanding of how each functional is constructed and what exact conditions it does or does not obey, as well as extensive numerical testing.

The gradient-dependence of each GGA is most easily visualized [19] in the spin-unpolarized case $\left(n_{\uparrow}=n_{\downarrow}=n / 2\right)$, where we may write

$$
\begin{aligned}
E_{x c}^{\mathrm{GGA}}[n / 2, & n / 2] \\
& =\int d^{3} r n(\mathbf{r}) \epsilon_{x}(n(\mathbf{r})) F_{x c}\left(r_{s}(\mathbf{r}), s(\mathbf{r})\right) .
\end{aligned}
$$

Here,

$$
\epsilon_{x}(n)=-3 k_{F} / 4 \pi
$$

is the exchange energy per particle for a uniform gas of density $n$,

$$
k_{F}=\left(3 \pi^{2} n\right)^{1 / 3}
$$

is the local Fermi wave vector, and $F_{x c}\left(r_{s}, s\right)$ is the enhancement factor over local exchange. For each GGA under discussion, we shall plot $F_{x c}$ as a func-

\begin{tabular}{|c|c|c|c|c|c|c|}
\hline \multirow[b]{2}{*}{ Metal } & \multicolumn{3}{|c|}{ Lattice constant (bohr) } & \multicolumn{3}{|c|}{ Bulk modulus (GPa) } \\
\hline & LSD & PW91 & Expt. & LSD & PW91 & Expt. \\
\hline $\mathrm{Li}(\mathrm{bcc})^{\mathrm{a}}$ & 6.36 & 6.51 & 6.57 & 15.0 & 13.4 & 13.0 \\
\hline $\mathrm{Na}(\mathrm{bcc})^{\mathrm{a}}$ & 7.65 & 7.97 & 7.98 & 9.2 & 7.1 & 7.4 \\
\hline$V(b c c)^{b}$ & 5.53 & 5.66 & 5.74 & 212 & 184 & 157 \\
\hline $\mathrm{Nb}(\mathrm{bcc})^{\mathrm{b}}$ & 6.14 & 6.25 & 6.24 & 189 & 167 & 170 \\
\hline
\end{tabular}
tion of the reduced density gradient

$$
s=|\nabla n| /\left(2 k_{F} n\right)
$$

TABLE |I

Properties of solid metals.

From [19].

${ }^{b}$ From [20]. 
TABLE III Spin-polarization energies of atoms (in eV), using analytic Hartree-Fock densities, as in [56].

\begin{tabular}{lccc}
\hline Atom & $\sum P_{n}^{2}$ & LSD & PW91 \\
\hline $\mathrm{H}(1 s \uparrow)^{1}$ & 1 & -0.98 & -1.18 \\
$\mathrm{Li}(2 s \uparrow)^{\dagger}$ & 1 & -0.24 & -0.27 \\
$\mathrm{Be}+1(2 s \uparrow)^{1}$ & 1 & -0.49 & -0.53 \\
$\mathrm{~B}(2 p \uparrow)^{1}$ & 1 & -0.26 & -0.28 \\
$\mathrm{~F}(2 p \uparrow)^{3}(2 p \downarrow)^{2}$ & 1 & -0.38 & -0.39 \\
$\mathrm{Sc}(4 s)^{2}(3 d \uparrow)^{1}$ & 1 & -0.18 & -0.19 \\
$\mathrm{C}(2 p \uparrow)^{2}$ & 4 & -1.22 & -1.28 \\
$\mathrm{O}(2 p \uparrow)^{3}(2 p \downarrow)^{1}$ & 4 & -1.43 & -1.48 \\
$\mathrm{Sc}(4 s \uparrow)^{1}(3 d \uparrow)^{2}$ & 5 & -0.99 & -1.10 \\
$\mathrm{~N}(2 p \uparrow)^{3}$ & 9 & -3.09 & -3.14 \\
\hline
\end{tabular}

Note that $\Delta E_{x c} \sim \sum P_{n}^{2}$, where $P_{n}$ is the net number of electron spins in a shell with principal quantum number $n$.

for several values of the local Wigner-Seitz radius,

$$
r_{s}=(4 \pi n / 3)^{-1 / 3}
$$

These plots permit a ready comparison [42] of one GGA with another and a check on many of the exact conditions [43] which a density functional should satisfy.

To illustrate why we consider only the spinunpolarized case, Table III shows spin-polarization energies,

$$
\Delta E_{x c}[n]=E_{x c}\left[n_{\uparrow}, n_{\downarrow}\right]-E_{x c}[n / 2 . n / 2],
$$

evaluated for several atoms and ions. Although GGA favors spin polarization more than does LSD, the difference is small. Thus, for many purposes, we may compare GGAs by comparing only their spin-unpolarized enhancement factors $F_{x c}\left(r_{s}, s\right)$.

For practical purposes, the most important exact conditions are those which constrain $F_{x c}\left(r_{s}, s\right)$ in the ranges of $r_{s}$ and $s$ that dominate systems and properties of physical interest. Typically, $r_{s} \lesssim 1$ and $s \leqq 1$ in the core of an atom, with the largest values of $s$ occurring in the outermost part of a shell of principal quantum number $n$, just before the next shell cuts in. Valence electrons in metals have $1 \leq r_{s} \leqslant 6$ and $s \leqslant 2$, with $s=0$ on the boundary of a unit cell. Moving out through the surface of a finite system, $r_{s}$ and $s$ do grow exponentially. However, these low-density regions make little contribution to the energy.

The focus of this article is a comparison of popular functionals on the basis of those formal properties which are important for real systems. In the next section, we discuss those conditions which constrain functionals in regions of $r_{s}$ and $s$ which dominate real systems. Other exact conditions are discussed in the third section. Then, in the fourth section, we consider the three most popular functionals for exchange, showing their substantial similarity. In the following section, we make a detailed graphical comparison of eight popular functionals for exchange and correlation. The sixth section is a discussion of the character of the gradient expansion and its generalization. We conclude in the final section with a summary of results for the shopper in a hurry.

\section{Exact Conditions for the Discriminating Shopper}

Every functional of the GGA form Eq. (2) is properly size-consistent, i.e., the energy of a system of well-separated fragments (e.g., separated atoms) is just the sum of the energies of the individual fragments, so that binding energy curves may be predicted. But no GGA is properly selfinteraction free [44], i.e., no $E_{x c}^{\mathrm{GGA}}$ can exactly cancel the Hartree electrostatic self-energy for every one-electron density.

Formally, the exchange-correlation energy is $[45,46]$

$$
E_{x c}=\frac{1}{2} \int d^{3} r n(\mathbf{r}) \int d^{3} r^{\prime} \frac{n_{x c}\left(\mathbf{r}, \mathbf{r}^{\prime}\right)}{\left|\mathbf{r}^{\prime}-\mathbf{r}\right|}
$$

where $n_{x c}\left(\mathbf{r}, \mathbf{r}^{\prime}\right)$ is the density at $\mathbf{r}^{\prime}$ of the exchange-correlation hole around an electron at $\mathbf{r}$, and $n_{x c}=n_{x}+n_{c}$. The exchange hole density arising from the Pauli exclusion principle is strictly negative:

$$
n_{x}\left(\mathbf{r}, \mathbf{r}^{\prime}\right) \leq 0
$$

and represents a deficit of exactly one electron:

$$
\int d^{3} r^{\prime} n_{x}\left(\mathbf{r}, \mathbf{r}^{\prime}\right)=-1
$$

The correlation hole arising from the Coulomb repulsion does not change the electron number:

$$
\int d^{3} r^{\prime} n_{c}\left(\mathbf{r}, \mathbf{r}^{\prime}\right)=0
$$


Another hole condition which has been thought to be exact [46] is

$$
n_{x c}(\mathbf{r}, \mathbf{r})=n_{x c}^{\mathrm{LSD}}(\mathbf{r}, \mathbf{r}) \text {. }
$$

Satisfaction of Eqs. (10)-(13) provides a powerful rationale $[45,47]$ for the LSD approximation and for certain GGAS. Recently, we showed [48] that Eq. (13) is not exact, except in the high-density, lowdensity, and fully spin-polarized limits. Nevertheless, Eq. (13) can be remarkably accurate for realistic electron densities.

For a uniform system, LSD is exact, i.e.,

$$
F_{x c}\left(r_{s}, s=0\right)=\epsilon_{x c}(n) / \epsilon_{x}(n) .
$$

For a slowly varying density $(s \ll 1)$, i.e., a density which varies little over the range of the exchange-correlation hole, which has radius $\approx r_{s}$, the second-order gradient expansion is exact, i.e.,

$$
\begin{aligned}
F_{x c}\left(r_{s}, s \ll 1\right)= & F_{x c}\left(r_{s}, s=0\right) \\
& +\left[\alpha_{x}+\alpha_{c}\left(r_{s}\right)\right] s^{2}+\ldots,
\end{aligned}
$$

where the gradient coefficients $\alpha_{x}$ and $\alpha_{c}\left(r_{s}\right)$ are known [5, 49-51]. In most GGAs, $F_{x c}\left(r_{s}, s\right)$ deviates strongly from Eq. (15) for $s \geq 0.1$; thus, recovery of the exact gradient coefficient $\alpha_{c}\left(r_{s}\right)$ as $s \rightarrow 0$ is not a very important condition for most practical purposes.

Coordinate scaling $[43,52-57]$ provides some powerful constraints. Define a uniform scaling of the density $n(\mathbf{r})$ by

$$
n_{\gamma}(\mathbf{r})=\gamma^{3} n(\gamma \mathbf{r}),
$$

for $\gamma>0$, so that the total number of electrons, $n=\int d^{3} m_{\gamma}(\mathbf{r})$, remains fixed for all $\gamma$. The fundamental scaling inequality is [52]

$$
E_{x c}\left[n_{\gamma}\right]>\gamma E_{x c}[n] ; \quad(\gamma>1),
$$

which implies, since $s_{\gamma}(\mathbf{r})=s(\gamma \mathbf{r})$,

$$
F_{x c}\left(r_{s}^{\prime}, s\right)>F_{x c}\left(r_{s}, s\right) ; \quad\left(r_{s}^{\prime}>r_{s}\right) .
$$

Moreover [52],

$$
E_{x}\left[n_{\gamma}\right]=\gamma E_{x}[n],
$$

i.e.,

$$
F_{x}\left(r_{s}, s\right)=F_{x}(s) .
$$

In the high-density limit $(\gamma \rightarrow \infty)$, we have [52]

$$
\lim _{\gamma \rightarrow \infty} \frac{1}{\gamma} E_{x c}\left[n_{\gamma}\right]=E_{x}[n]
$$

i.e.,

$$
\lim _{r_{s} \rightarrow 0} F_{x c}\left(r_{s}, s\right)=F_{x}(s) .
$$

Since $F_{c}\left(r_{s}=0, s\right)=0$, Eqs. (18) and (22) imply that

$$
F_{c}\left(r_{s}, s\right)>0 .
$$

Furthermore [53, 54],

$$
\lim _{\gamma \rightarrow \infty} E_{c}\left[n_{\gamma}\right]>-\infty .
$$

These conditions are important, because they constrain $F_{x c}\left(r_{s}, s\right)$ in the dominant range of small or moderate $r_{s}$ and $s$.

\section{Other Exact Conditions}

Some exact conditions on $E_{x c}$ are satisfied only when $F_{x c}\left(r_{s}, s\right)$ is constrained at large values of $r_{s}$ and $s$. For example, the Lieb-Oxford bound [15, $58]$ is satisfied if

$$
F_{x c}\left(r_{s}, s\right) \leq 2.27,
$$

and various nonuniform scaling relations [53-55] are satisfied only if [43]

$$
\lim _{s \rightarrow \infty} s^{1 / 2} F_{x}\left(r_{s}, s\right)<\infty
$$

and

$$
\lim _{s \rightarrow \infty} s^{1 / 2} F_{c}\left(r_{s}, s\right)<\infty .
$$

However, Lacks and Gordon [59] found that the interaction between rare-gas atoms at large separation was better modeled by $F_{x c} \approx s^{2 / 5}$ at large $s$. Becke [11] matched the asymptotic $(r \rightarrow \infty)$ exchange energy density of an atom with $F_{x} \approx s / \ln s$, while Engel et at. [60] matched the asymptotic exchange potential $(-1 / r)$ with $F_{x} \approx s$. In fact, the GGA form itself [Eq. (2)] fails for $s \gg 1$, so it is not surprising that various exact conditions imply contradictory large $s$ limits for $F_{x c}\left(r_{s}, s\right)$.

We also note that the correct $r \rightarrow \infty$ limit for the exchange-correlation potential $v_{x c, \sigma}(\mathbf{r})$ of an atom is not $-1 / r$, but $-1 / r+C / 2$, where $C$ is a positive constant, as a result of averaging over the discontinuity in the exact Kohn-Sham potential when the electron number crosses an integer [61, 
62]. LSD and GGA are continuum approximations; they include an estimate of the constant $C / 2$ in their exchange-correlation potentials in the interior of the atom, but break down as $r \rightarrow \infty$. Whenever the ground states of the positive and negative ions can be found by removing or adding an electron in the highest occupied Kohn-Sham energy level of the neutral atom, the constant $C$ is just the difference between the ionization energy and the electron affinity of the atom. To take boron as an example, $C / 2=4.0 \mathrm{eV}$ accounts for the difference between the LSD and GGA $2 p \uparrow$ orbital energy and minus the ionization energy.

For many atoms, the optimized local exchange potential $v_{x, \sigma}(\mathbf{r})$ is known [63-65], and $v_{x, \sigma}(\mathbf{r}) \rightarrow$ $-1 / r$ as $r \rightarrow \infty$. Some GGAs have been constructed $[66,67]$ by fitting $\delta E_{x} / \delta n_{\sigma}(\mathbf{r})$ to $v_{x, \sigma}(\mathbf{r})$, but we suggest, in light of the previous paragraph, that it would be better to fit $\nabla \delta E_{x} / \delta n_{\sigma}(\mathbf{r})$ to $\nabla v_{x, \sigma}(\mathrm{r})$.

Finally, we note that the low-density limit

$$
A[n]=\lim _{\gamma \rightarrow 0} \frac{1}{\gamma} E_{x c}\left[n_{\gamma}\right]+\frac{1}{2} \int d^{3} r \int d^{3} r^{\prime} \frac{n(\mathbf{r}) n\left(\mathbf{r}^{\prime}\right)}{\left|\mathbf{r}-\mathbf{r}^{\prime}\right|}
$$

has been shown to be a convex functional [43], i.e.,

$$
A\left[d_{1} n_{1}+d_{2} n_{2}\right] \leq d_{1} A\left[n_{1}\right]+d_{2} A\left[n_{2}\right],
$$

where $n_{i} \geq 0, \int d^{3} r n_{i}(\mathbf{r})=N, d_{i} \geq 0$, and $d_{1}+$ $d_{2}=1$. This is a severe constraint which GGAs are probably unable to obey [42] and provides a challenge for the approximate functionals of the future.

\section{Exchange Energy}

The local spin density (LSD) approximation for exchange,

$$
F_{x}^{\mathrm{LSD}}(\mathrm{s})=1,
$$

satisfies Eqs. (10), (11), (13), (14), (20), and (25), but violates conditions Eqs. (15) and (26).

The second-order gradient expansion approximation [51] (GEA),

$$
F_{x}^{\mathrm{GEA}}(s)=1+0.1234 s^{2},
$$

additionally satisfies Eq. (15), but violates Eq. (26), and, more importantly, the hole conditions Eqs. (10) and (11). Nevertheless, GEA is typically an improvement over LSD for the exchange energy of an atom.
The GEA exchange hole density $n_{x}(\mathbf{r}+\mathbf{u}, \mathbf{r})$ is reasonable for small values of the interelectronic separation $u=|\mathbf{u}|$. But, for large values of $u$, the GEA hole displays $[9,10,68]$ an undamped $\cos \left(2 k_{F} u\right)$ oscillation which satisfies the hole normalization condition of Eq. (11) only with the help of a convergence factor.

To cure these pathologies, Perdew and Wang [9] devised the real-space cutoff procedure, in which the spurious long-range part of the GEA hole density is sharply cut off to restore the constraints of Eqs. (10) and (11). This real-space cutoff procedure follows naturally from a real-space analysis of $E_{x c}$ [69]. The result is a numerically defined nonempirical function $F_{x}(s)$. This function was subsequently fitted to an analytic form, PW86,

$$
F_{x}^{\mathrm{PW} 86}(s)=\left(1+1.296 s^{2}+14 s^{4}+0.2 s^{6}\right)^{1 / 15}
$$

Thus, with no empirical input, the PW86 functional reduced the error in the calculated exchange energies of atoms from the LSD level of $10 \%$ or less to $1 \%$ or less. (Incidentally, as discussed in the previous section, Pw86 also happens to give a good account of the interaction between rare-gas atoms at large separation [59].)

Subsequently, Becke [11] proposed a GGA for the exchange energy, B88, that reproduces the exact asymptotic $(r \rightarrow \infty)$ behavior of the exchange energy density and depends on a single parameter which was adjusted to minimize the error in the exchange energies of the rare-gas atoms. This functional is at least as successful as is PW86, despite the fact that the gradient coefficient $\alpha_{x}$ of Eq. (15) is considerably overestimated by the B88 functional, thereby demonstrating the relative unimportance of satisfying Eq. (15).

More recently, Perdew and Wang $[15,16,19]$ noted that both the PW86 and B88 functionals violated Eqs. (25) and (26). Starting from Becke's form, they modified the large $s$ behavior to satisfy Eqs. (25) and (26) and the small $s$ behavior to recover Eq. (31).

In the important range $0<s<2$, the Pw86, B88, and PW91 enhancement factors are not very different, as will be apparent from a study of the $r_{s}=0$ curves for PW86, BP, and PW91 in the next section. Although PW91 is designed to satisfy the largest number of "universal" conditions valid for all electronic densities, there is no compelling formal reason to prefer one functional over the other. The 
differences between these functionals are probably smaller than is the error made in using the GGA form [Eq. (2)]. Thus, the real-space cutoff procedure $[8,9]$ may be considered as a justification for all three of these functionals.

\section{Exchange-Correlation Energy}

The first GGA for the correlation energy was proposed by Ma and Brueckner [5], who observed that the unmodified GEA unfortunately gave positive correlation energies for atoms. Since then, a number of GGAS have been proposed for $E_{c}$.

It is possible to treat the exchange energy exactly, as in Hartree-Fock theory, leaving only the correlation energy to be approximated by a GGA [70-73]. Numerical results for atoms and molecules are typically better than for Hartree-Fock alone, but not so good as when GGA is used for exchange and correlation together. The atomization energy of $\mathrm{C}_{2}$ in Table $\mathrm{I}$ is a particularly striking example [19] of a cancellation of error between GGA exchange (X) and correlation (C): The GGA-XC atomization energy of $6.55 \mathrm{eV}$ is close to the experimental value of $6.36 \mathrm{eV}$, but the $\mathrm{GGA}^{-} \mathrm{X}$ atomization energy of $5.0 \mathrm{eV}$ is far from the Hartree-Fock value of $0.73 \mathrm{eV}$.

Because the exchange-correlation hole around an electron is deeper and more localized than is the exchange hole, LSD and GGA are better approximations for exchange and correlation together than

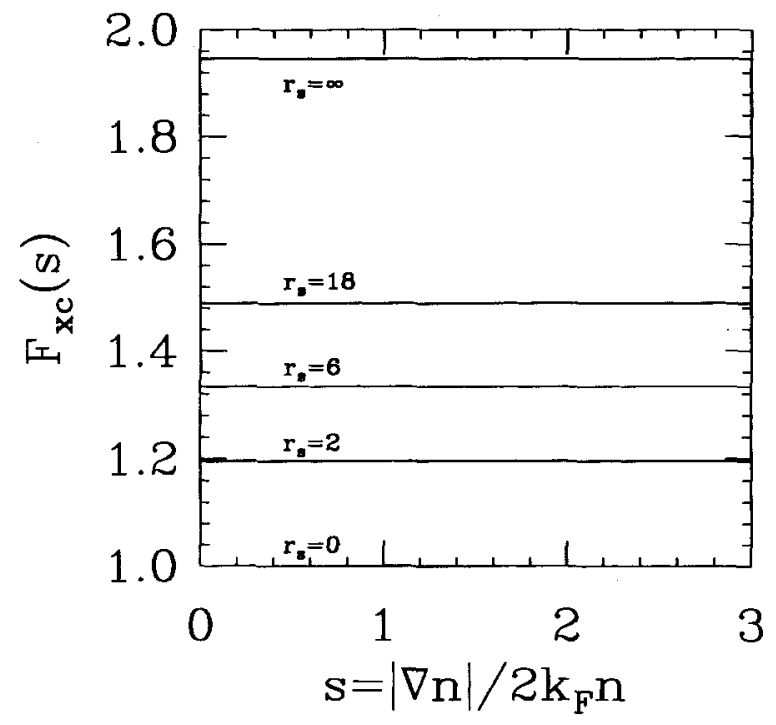

FIGURE 1. $F_{x c}(s)$ for LSD.

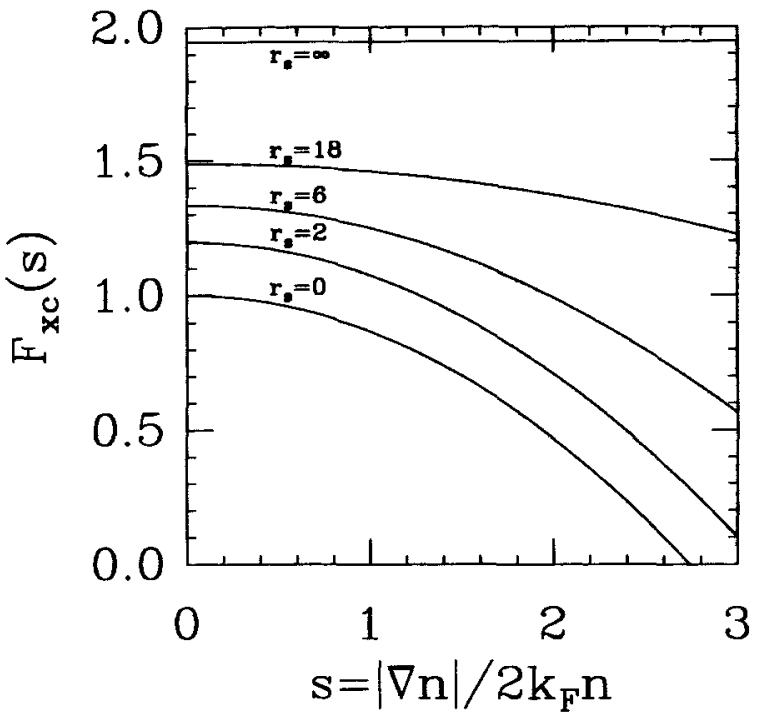

FIGURE 2. $F_{x c}(s)$ for GEA.

for either separately. Thus, we display $F_{x c}\left(r_{s}, s\right)$ in the following figures.

Figure 1 is a plot of $F_{x c}$ in the LSD approximation [1, 41] of Eq. (14). The curves are horizontal lines, as the LSD approximation is independent of the local density gradient. In the high-density limit $\left(r_{s}=0\right)$, where exchange dominates, $F_{x c}^{\mathrm{LSD}}\left(r_{s}, s\right)=$ 1. The increase in $F_{x c}^{\mathrm{LSD}}$ beyond 1 for finite values of $r_{s}$ represents the correlation contribution. Because the LSD hole is the hole of a possible physical system (the uniform electron gas), the LSD approximation respects Eqs. (10)-(14), (18), (22), and (25). However, LSD violates Eqs. (15), (24), (26), and (27).

The gradient expansion approximation [50] (GEA), Eq. (15) to second-order in $s$, does not fare so well. Its exchange-correlation hole is not the hole of any physical system and so violates the hole conditions [Eqs. (10)-(12)]. In particular, the correlation hole integrates not to zero, but to a positive number. Figure 2 shows the parabolic curves $F_{x c}^{\mathrm{GEA}}\left(r_{s}, s\right)$, which do not even reduce to $F_{x}^{\mathrm{GEA}}(s)$ when $r_{s} \rightarrow 0$, as they should according to Eq. (22). While GEA is an improvement over LSD for slowly varying densities ( $s \ll 1$ everywhere), it is typically worse than LSD for real electronic systems.

The first of the modern GGAs was proposed by Langreth and Mehl [6], as shown in Figure 3. This functional was constructed from a decomposition [74] of $E_{x c}$ into contributions from various wave vectors $\mathbf{k}$ of dynamic density fluctuations within the random-phase approximation (RPA). (This wave 


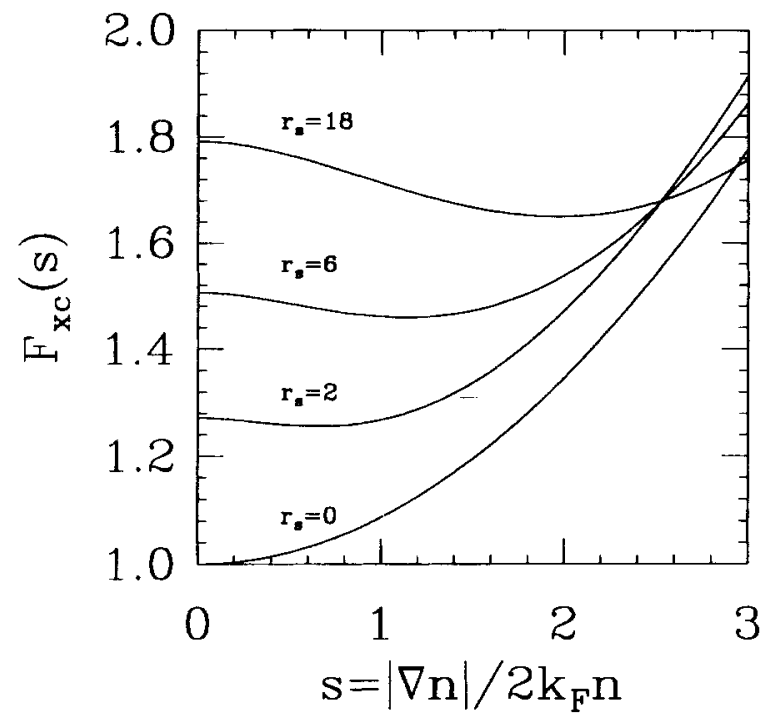

FIGURE 3. $F_{x c}(s)$ for $L M$.

vector is the Fourier transform variable of the interelectronic separation u.) Essentially, the full GEA for exchange was retained by LM, but the spurious small $k$ (large $u$ ) contribution to the gradient term in the correlation energy was replaced by zero for $k<f|\nabla n| / n$, where the cutoff parameter $f=0.15$ was adjusted to provide an overall fit to the correlation energies of atoms and metal surfaces; $f \approx 1 / 6$ had been expected on theoretical grounds. Figure 3 shows that LM cures the worst error of GEA, by turning off the correlation contri-

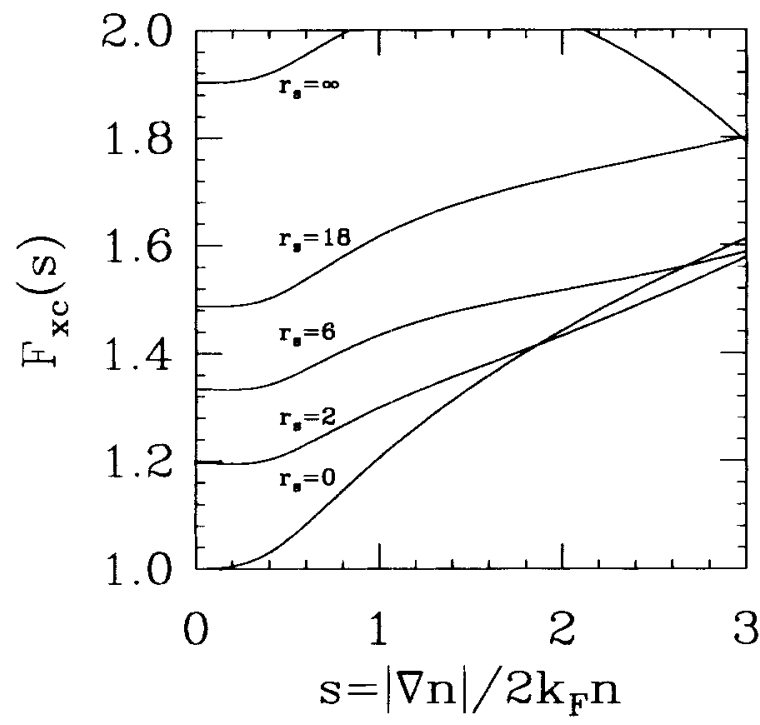

FIGURE 4. $F_{x c}(s)$ for Pw86. bution to $F_{x c}$ in the limit $r_{s} \rightarrow 0$. However, the curve-crossing in Figure 3 does violate the Eq. (18). Moreover, because the LM functional is based on RPA, it does not recover the correct uniform gas limit of Eq. (14).

The Perdew-Wang 1986 functional of Figure 4 combines Pw86 exchange [9] with the Perdew 1986 [10] correlation energy functional. The latter was constructed via a wave vector cutoff procedure similar to that of LM, but with beyond-RPA inputs for the uniform and slowly varying electron gases. Since Eq. (12) is satisfied by any choice of the cutoff wave vector $f|\nabla n| / n$, where $f>0$, the parameter $f$ was found by fitting the correlation energy of the neon atom. PW86 obeys the exact conditions of Eqs. (14) and (15). It still has curvecrossing problems, but not as severe as those of LM. It violates Eqs. (25)-(27) and Eq. (23). Sometimes, Becke exchange [11] is combined with Perdew 86 [10] correlation to form the BP approximation, shown in Figure 5, which is similar to PW86, as discussed in the previous section.

A more recent correlation energy functional is that of Lee, Yang, and Parr [12], which is often used in conjunction with $\mathrm{B} 88$ for exchange [11] to form BLYP. The LYP functional starts from the Colle-Salvetti formula for the correlation energy in terms of the electron density and the noninteracting kinetic energy density, then replaces the latter by its second-order density-gradient expansion. (The Colle-Salvetti formula itself is derived from a number of theoretical approximations

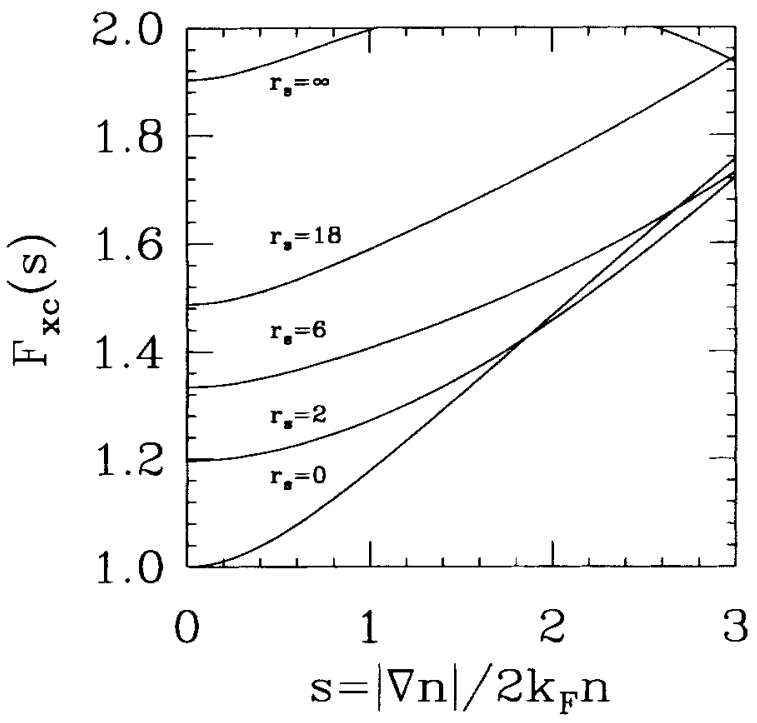

FIGURE 5. $F_{x c}(s)$ for $\mathrm{BP}$. 


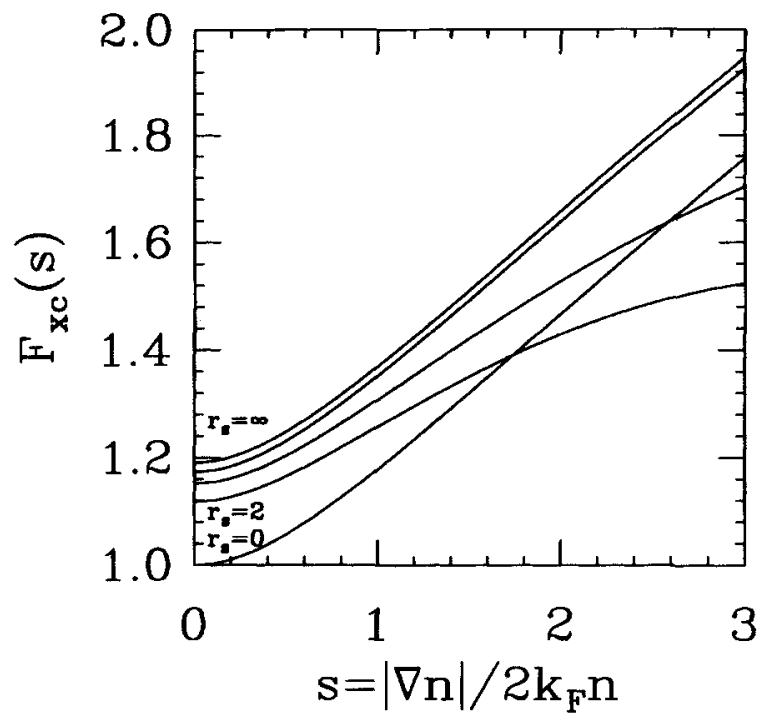

FIGURE 6. $F_{x c}(s)$ for BLYP; the two unlateled curves are for $r_{s}=6$ (lower) and $r_{s}=18$ (higher).

and is fitted to the correlation energy of the helium atom.) The result is then cast into the GGA form of Eq. (2) via integration by parts [75] and plotted in Figure 6. Clearly, BLYP is inaccurate in the uniform limit ( $s=0$ ), i.e., Eq. (14) is violated. BLYP also violates Eqs. (15), (18), and (25)-(27), but notably obeys Eq. (24): It makes the correlation energy scale to a constant in the high-density limit.

Becke exchange may also be combined with Wilson-Levy [13] (WL) correlation to form BWL,

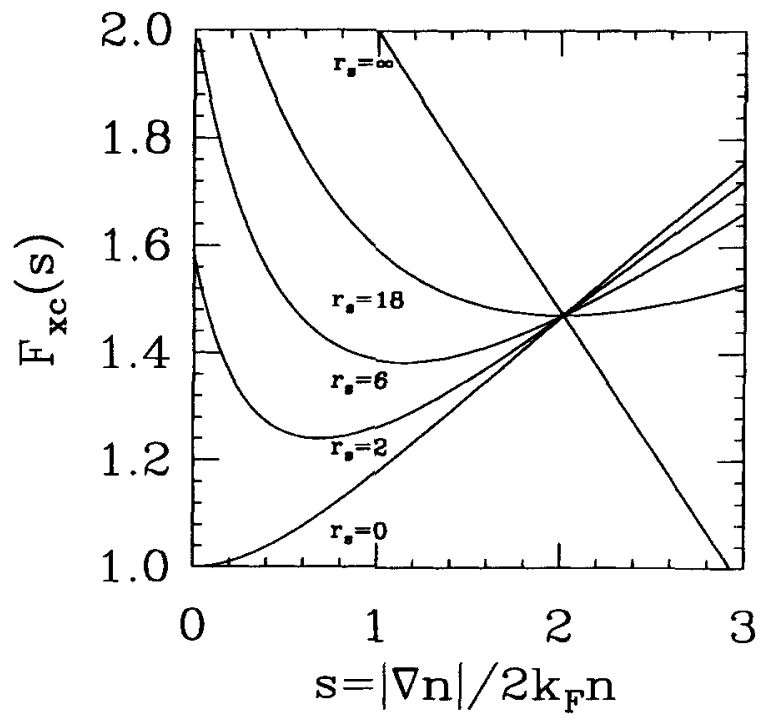

FIGURE 7. $F_{x c}(s)$ for BWL.

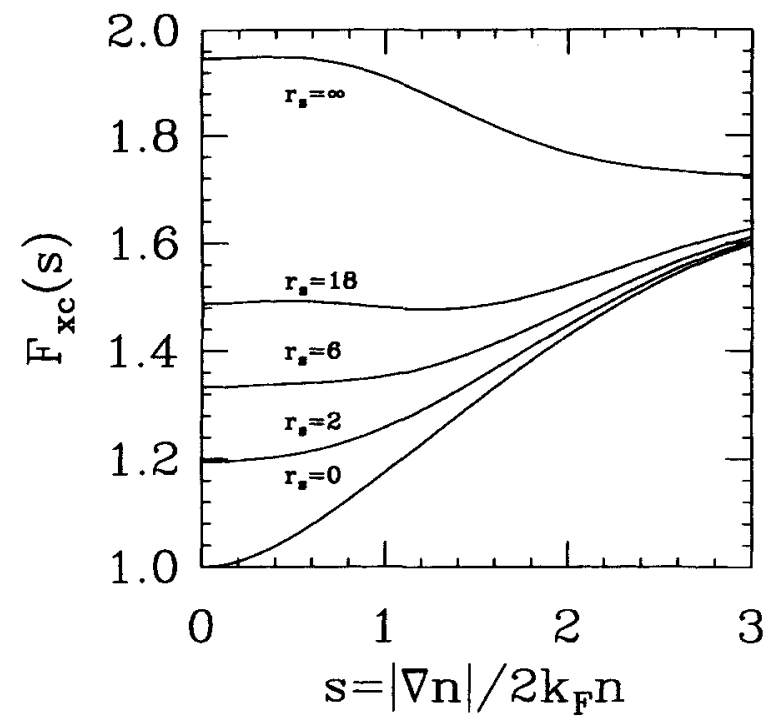

FIGURE 8. $F_{x c}(s)$ for Pw91.

shown in Figure 7. The wL correlation functional was designed to satisfy a few, but not all, of the scaling relations that were known at the time of its construction. Starting from a Wigner-like form with gradient corrections, the coefficients in this functional were adjusted to minimize $\left[\partial E_{\mathrm{c}}\left[n_{\gamma}\right] / \partial \gamma\right]^{2}$ for eight closed-shell atoms, while fitting the correlation energy of the He atom. We see that BWL is inaccurate in the uniform $(s=0)$ and slowly varying $(s \ll 1)$ limits; in the latter limit, it does not even reduce to the form of Eq. (15). WL also violates Eqs. (18) and (25), but, like BLYP, it respects Eq. (24).

Like LSD and GEA, and unlike the other GGAS discussed here, the Perdew-Wang $91[14-16,19]$ (PW91) functional, shown in Figure 8, is nonempirical, in the sense that it was constructed using only the gradient expansion for the hole density plus the exact conditions of the second and third sections. The construction of the PW91 exchange energy was discussed in the previous section. The PW92 correlation energy is also constructed via a sharp real-space cutoff of the spurious long-range part of the gradient expansion for the hole density, with the cutoff radius chosen to obey Eq. (12). Unlike the exchange part, the correlation part of PW91 is taken directly from the real-space cutoff, with no further modification. This leads naturally to satisfaction of Eq. (27). In fact, it is clear from Figure 8 that PW91 correlation "turns off" as the reduced density gradient $s$ increases. 
Of all the density functionals discussed here, PW91 best satisfies the formal properties of the second and third sections. In fact, it satisfies all of the numbered exact conditions, except Eq. (24), which it nearly satisfies, and the convexity condition of Eq. (28), which no GGA is known to satisfy. (At unrealistically high densities and small gradients, PW91 also violates [76] Eq. (23). With a very slight modification proposed by Levy and Perdew [43], PW91 can be made to satisfy Eqs. (23) and (24).)

Comparison of Figures 1-8 shows that PW91 is "more local" (i.e., more like LSD) than any of the other GGAS. Also, it becomes increasingly more local as the density is reduced. The explanation for the latter behavior is that, as $r_{s}$ increases from 0 to $\infty$, the hole density at $\mathbf{u}=\mathbf{0}$ drops from its highdensity limit of $-n / 2$ to its low-density limit of $-n$, and, because it integrates to -1 , the hole becomes more localized on the scale of $r_{s}$. This explains why LSD works as well as it does and also why it works better for exchange and correlation together than for exchange alone.

\section{Character of the Gradient Expansion and Its Generalization}

The first-principles density-gradient expansion of the system-averaged exchange-correlation hole,

$$
\left\langle n_{x c}(\mathbf{u})\right\rangle=\frac{1}{N} \int d^{3} r n(\mathbf{r}) n_{x c}(\mathbf{r}, \mathbf{r}+\mathbf{u}),
$$

is known $[15,16]$ only to second order in $\nabla$ and constitutes an input into PW91. The exchange hole $n_{x}(\mathbf{r}, \mathbf{r}+\mathbf{u})$ is known [77] to third order in $\nabla$. The noninteracting kinetic energy, constructed [78] from the Taylor expansion of $n_{x}(\mathbf{r}, \mathbf{r}+\mathbf{u})$ to second order in $\mathbf{u}$, is known [79] to sixth order in $\nabla$.

Based on this limited knowledge, the gradient expansion seems to have the following character [77]: In a system of slowly varying electron density $n(\mathbf{r})$, the addition of each successive term of higher order in $\nabla$ improves the description of the hole close to its electron (small $u$ ), but worsens the description of the hole far away (large $u$ ). In such a system, the kinetic energy has a rapidly convergent gradient expansion. The exchange-correlation energy

$$
E_{x c}=\frac{N}{2} \int d^{3} u \frac{\left\langle n_{x c}(\mathbf{u})\right\rangle}{u}
$$

would also have a rapidly convergent gradient expansion if the electron-electron interaction were short-ranged, but the Coulomb interaction, $1 / u$, foils this expectation.

If we define a set of reduced density derivatives, such as $s$ in Eq. (6), or

$$
t=|\nabla n| /\left(2 k_{s} n\right),
$$

where $k_{s}=\left(4 k_{F} / \pi\right)^{1 / 2}$ is the Thomas screening length, or $\nabla^{2} n /\left[\left(2 k_{F}\right)^{2} n\right]$, etc., we may imagine a system in which all such reduced derivatives are well bounded. A bulk solid is a realistic example of such a system, apart from the cusps in the electron density, which may be removed by pseudopotential theory. Even in such a system, we should not expect that the gradient expansion for $E_{x c}$ converges as terms of higher order in $\nabla$ are added, although we know it gives the right asymptotic expansion in the limit where all reduced density derivatives are small. However, in each order we may construct a generalized gradient expansion by cutting off the spurious large $u$ contributions to $n_{x c}$ in a way that respects Eqs. (10)-(12). We have no reason to doubt that this sequence will converge. The first member of this sequence is LSD, and the next, apart from minor ambiguities in the selection of a cutoff procedure, etc., is the PW91 generalized gradient approximation. (Note that the noninteracting kinetic energy is unaffected [80] by real-space cutoffs at finite values of $u$.)

These considerations suggest that pseudopotential models of bulk solids may be the most refined future testing grounds for gradient expansions and generalized gradient approximations against more intensive calculations. For these systems, Hartree-Fock results $[72,81]$ are already available for testing kinetic and exchange energy functionals, and quantum Monte Carlo results [82] have begun to appear for testing exchange-correlation energy functionals. However, Kohn-Sham theory [1] requires that the external potential to be local (i.e., multiplication) operator $V(\mathbf{r})$, while most pseudopotentials are nonlocal [18, 29, 83-85] or angular momentum dependent operators $V\left(\mathbf{r}, \mathbf{r}^{\prime}\right)$. Only recently have realistic, nonempirical, local pseudopotentials been constructed [86] for solids.

It should be noted that even very realistic pseudopotentials can introduce subtle errors of their own. Thus, for comparisons between GGA and experimental results for solids or other systems, we recommend careful all-electron, full-potential cal- 
culations like those of $[19,20,87,88]$. (The cusps of the all-electron density should not significantly affect bond lengths, vibration frequencies, or atomization energies.)

\section{Conclusions}

Comparison shopping among various generalized gradient approximations for the exchangecorrelation energy, on the basis of known exact formal conditions, leads to the following conclusions: Among exchange functionals, the PW86, B88, and PW91 brands are about equally good. Among correlation functionals, the nonempirical PW91 brand seems the best. Its construction is based on an (albeit speculative) insight into the character of the gradient expansion and its generalization. Subroutines which evaluate the PW91 exchangecorrelation energy density and potential from the electron spin densities and their derivatives are available gratis by electronic mail from perdew@mailhost.tcs.tulane.edu.

While improving atomization energies over LSD (Table I), PW91 remains the most "local" of the gradient corrected exchange-correlation functionals, as seen in Figures 1-8, and thus the least likely to overcorrect the subtle errors of LSD for solids (Table II). Of course, the ultimate test is in the marketplace, so we have recently reviewed [42] many comparisons of GGA predictions with experimental or exact results over a wide range of real or model systems.

\section{ACKNOWLEDGMENTS}

This work has been supported by NSF Grant No. DMR92-13755.

\section{References}

1. W. Kohn and L. J. Sham, Phys. Rev. A 140, 1133 (1965).

2. R. G. Parr and W. Yang, Density Functional Theory of Atoms and Molecules (Oxford, New York, 1989).

3. R. M. Dreizler and E. K. U. Gross, Density Functional Theory (Springer-Verlag, Berlin, 1990).

4. R. O. Jones and O. Gunnarsson, Rev. Mod. Phys. 61, 689 (1989).

5. S. K. Ma and K. A. Brueckner, Phys. Rev. 165, 18 (1968).

6. D. C. Langreth and M. J. Mehl, Phys. Rev. B 28, 1809 (1983).

7. C. D. Hu and D. C. Langreth, Phys. Scr. 32, 391 (1985).
8. J. P. Perdew, Phys. Rev. Lett. 55, 1665 (1985); Ibid. 55, 2370 (1985).

9. J. P. Perdew and Y. Wang, Phys. Rev. B 33, 8800 (1986); Ibid. 40, 3399 (1989).

10. J. P. Perdew, Phys. Rev. B 33, 8822 (1986); Ibid. 34, 7406 (1986).

11. A. D. Becke, Phys. Rev. A 38, 3098 (1988).

12. C. Lee, W. Yang, and R. G. Parr, Phys. Rev. B 37, 785 (1988).

13. L. C. Wilson and M. Levy, Phys. Rev. B 41, 12930 (1990).

14. J. P. Perdew, Physica B 172, 1 (1991).

15. J. P. Perdew, in Electronic Structure of Solids '91, P. Ziesche and H. Eschrig, Eds. (Akademie Verlag, Berlin, 1991).

16. J. P. Perdew, K. Burke, and Y. Wang, unpublished.

17. P. Bagno, O. Jepsen, and O. Gunnarsson, Phys. Rev. B 40, 1997 (1989).

18. G. Ortiz and P. Ballone, Phys. Rev. B 43, 6376 (1991).

19. J. P. Perdew, J. A. Chevary, S. H. Vosko, K. A. Jackson, M. R. Pederson, D. J. Singh, and C. Fiolhais, Phys. Rev. B 46, 6671 (1992); Ibid. 48, 4978 (1993).

20. V. Ozolins and M. Körling, Phys. Rev. B 48, 18304 (1993).

21. P. Mlynarski and D. R. Salahub, J. Chem. Phys. 95, 6050 (1991).

22. A. D. Becke, J. Chem. Phys. 96, 2155 (1992).

23. A. D. Becke, J. Chem. Phys. 97, 9173 (1992).

24. J. Andzelm and E. Wimmer, J. Chem. Phys. 96, 1280 (1992).

25. F. W. Kutzler and G. S. Painter, Phys. Rev. B 45, 3236 (1992).

26. R. Merkle, A. Savin, and H. Preuss, Chem. Phys. Lett. 194, 32 (1992).

27. J. M. Seminario, Chem. Phys. Lett. 206, 547 (1993).

28. B. G. Johnson, P. M. W. Gill, and J. A. Pople, J. Chem. Phys. 98, 5612 (1993).

29. H. Chen, M. Krasowski, and G. Fitzgerald, J. Chem. Phys. 98, 8710 (1993).

30. C. W. Murray, N. C. Handy, and R. D. Amos, J. Chem. Phys. 98, 7145 (1993).

31. A. Stirling, I. Pápai, J. Mink, and D. R. Salahub, J. Chem. Phys. 100, 2910 (1994).

32. B. Hammer, K. W. Jacobsen, and J. K. Nørskov, Phys. Rev. Lett. 70, 3971 (1993).

33. C. Sosa and C. Lee, J. Chem. Phys. 98, 8004 (1993).

34. R. V. Stanton and K. M. Merz, J. Chem. Phys. 100, 434 (1994).

35. K. Laasonen, F. Csajka, and M. Parinello, Chem. Phys. Lett. 194, 172 (1992).

36. C. Lee, D. Vanderbilt, K. Laasonen, R. Car, and M. Parrinello, Phys. Rev. Lett. 69, 462 (1992).

37. R. N. Barnett and U. Landman, Phys. Rev. Lett. 70, 1775 (1993).

38. J. M. Seminario, Int. Journal Quantum Chem. S 28, 655 (1994).

39. L. Fan, and T. Ziegler, J. Chem. Phys. 95, 7401 (1991).

40. S. H. Vosko, L. Wilk, and M. Nusair, Can. J. Phys. 58, 1200 (1980).

41. J. P. Perdew and Y. Wang, Phys. Rev. B 45, 13244 (1992).

42. K. Burke, J. P. Perdew and M. Levy, in Modern Density Functional Theory: A Tool for Chemistry, J. M. Seminario and P. Politzer, Eds. (Elsevier, Amsterdam, 1995). 
43. M. Levy and J. P. Perdew, Phys. Rev. B 48, 11638 (1993).

44. J. P. Perdew and A. Zunger, Phys. Rev. B 23, 5048 (1981).

45. O. Gunnarsson and B. I. Lundqvist, Phys. Rev. B 13, 4274 (1976).

46. D. C. Langreth and J. P. Perdew, Phys. Rev. B 15, 2884 (1977).

47. J. Harris, Phys. Rev. A 29, 1648 (1984).

48. K. Burke, J. P. Perdew, and D. C. Langreth, Phys. Rev. Lett. 73, 1283 (1994).

49. M. Rasolt and H. L. Davis, Phys. Lett. A 86, 45 (1981).

50. M. Rasolt and D. J. W. Geldart, Phys. Rev. B 34, 1325 (1986).

51. L. Kleinman and S. Lee, Phys. Rev. B 37, 4634 (1988).

52. M. Levy and J. P. Perdew, Phys, Rev. A 32, 2010 (1985).

53. M. Levy, Int. J. Quantum Chem. S23, 617 (1989).

54. M. Levy, Phys. Rev. A 43, 4637 (1991).

55. A. Görling and M. Levy, Phys. Rev. A 45, 1509 (1992).

56. A. Görling, M. Levy, and J. P. Perdew, Phys. Rev. B 47, 1167 (1993).

57. M. Levy, Bull. Am. Phys. Soc. 39, 671 (1994).

58. E. H. Lieb and S. Oxford, Int. J. Quantum Chem. 19, 427 (1981).

59. D. J. Lacks and R. G. Gordon, Phys. Rev. A 47, 4681 (1993).

60. E. Engel, J. A. Chevary, L. D. MacDonald, and S. H. Vosko, Z. Phys. D 23, 7 (1992).

61. J. P. Perdew, R. G. Parr, M. Levy and J. L. Balduz, Jr., Phys. Rev. Lett. 49, 1691 (1982).

62. J. P. Perdew, in Density Functional Methods in Physics, R. M. Dreizler and J. da Providencia, Ed. (Plenum, New York, 1985).

63. K. Aashamar, T. M. Luke, and J. D. Talman, Phys. Rev. A 19, 6 (1979).

64. V. Sahni, J. Gruenebaum, and J. P. Perdew, Phys. Rev. B 26, 4371 (1982).

65. J. B. Krieger, Y. Li, and G. J. Iafrate, in Density Functional Theory, R. Dreizler and E. K. U. Gross, Eds., NATO ASI Series (Plenum, New York, 1994).

66. S. H. Vosko and L. D. MacDonald, in Condensed Matter Theories, P. Vashishta, R. K. Kalia, and R. F. Bishop, Eds. (Plenum, New York, 1987), Vol. 2.
67. R. van Leeuwen and E. J. Baerends, Phys. Rev. A 49, 2421 (1994).

68. E. K. U. Gross and R. M. Dreizler, Z. Phys. A 302, 103 (1981).

69. K. Burke and J. P. Perdew, in Thirty Years of Density Functional Theory, 13-16 June, 1994, Cracow, Int. J. Quantum Chem., to appear.

70. E. Clementi and S. J. Chakravorty, J. Chem. Phys. 93, 2591 (1990).

71. P. Fuentealba and A. Savin, Chem. Phys. Lett. 217, 566 (1994).

72. M. Causá and A. Zupan, Chem. Phys. Lett. 220, 145 (1994).

73. N. Oliphant and R. J. Bartlett, J. Chem. Phys. 100, 6550 (1994).

74. D. C. Langreth and J. P. Perdew, Phys. Rev. B 21, 5469 (1980).

75. B. Miehlich, A. Savin, H. Stoll, and H. Preuss, Chem. Phys. Lett. 157, 200 (1989).

76. C. Umrigar and X. Gonze, in High Performance Computing and Its Application to the Physical Sciences, D. A. Browne et al., Eds. (World Scientific, Singapore, 1993).

77. Y. Wang, J. P. Perdew, J. A. Chevary, L. D. MacDonald, and S. H. Vosko, Phys. Rev. A 41, 78 (1990).

78. A. D. Becke, Int. J. Quantum Chem. 23, 1915 (1983).

79. D. R. Murphy, Phys. Rev. A 24, 1682 (1981).

80. J. P. Perdew, Phys. Lett. A 165, 79 (1992).

81. M. Causà, R. Dovesi, and C. Roetti, Phys. Rev. B 43, 11937 (1991).

82. L. Mitáš and R. M. Martin, Phys. Rev. Lett. 72, 2438 (1994).

83. A. Garcia, C. Elsässer, J. Zhu, S. G. Louie, and M. L. Cohen, Phys. Rev. B 46, 9829 (1992); lbid, 47, 4130 (1993).

84. Y. M. Juan and E. Kaxiras, Phys. Rev. B 48, 14944 (1993).

85. N. A. W. Holzwarth and Y. Zeng, Phys. Rev. B 49, 2351 (1994).

86. C. Fiolhais, J. P. Perdew, S. Q. Armster, J. M. MacLaren, and M. Brajczewska, unpublished.

87. P. Dufek, P. Blaha, V. Sliwko, and K. Schwarz, Phys. Rev. B 49, 10170 (1994).

88. R. Ahuja, S. Auluck, P. Söderlind, O. Eriksson, J. M. Willis, and B. Johansson, Phys. Rev. B 50, 11,183 (1994). 